\title{
In situ real-time annealing of ultrathin vertical Fe nanowires grown by focused electron beam induced deposition
}

\author{
Javier Pablo-Navarro ${ }^{1,2}$, Robert Winkler ${ }^{3}$, Georg Haberfehlner ${ }^{4}$, César Magén ${ }^{1,2,5}$, \\ Harald Plank ${ }^{3,4,6}$ and José María de Teresa ${ }^{1,2,5^{*}}$
}

${ }^{1}$ Laboratorio de Microscopías Avanzadas (LMA) - Instituto de Nanociencia de Aragón (INA), Universidad de Zaragoza, 50018 Zaragoza, Spain.

${ }^{2}$ Instituto de Ciencia de Materiales de Aragón (ICMA), Universidad de Zaragoza-CSIC, 50009 Zaragoza, Spain.

${ }^{3}$ Christian Doppler Laboratory DEFINE, Institute of Electron Microscopy and Nanoanalysis, Graz University of Technology, Steyrergasse 17, 8010 Graz, Austria.

${ }^{4}$ Institute of Electron Microscopy and Nanoanalysis, Graz University of Technology, Steyrergasse 17, 8010 Graz, Austria.

${ }^{5}$ Departamento de Física de la Materia Condensada, Universidad de Zaragoza, 50009 Zaragoza, Spain.

${ }^{6}$ Graz Centre for Electron Microscopy, Steyrergasse 17, 8010 Graz, Austria.

E-mail address of each author:

Javier Pablo-Navarro (javierpablonavarro@gmail.com)

Robert Winkler (robert.winkler@felmi-zfe.at)

Georg Haberfehlner (georg.haberfehlner@felmi-zfe.at)

César Magén (cmagend@unizar.es)

Harald Plank (harald.plank@felmi-zfe.at)

José María de Teresa (deteresa@unizar.es)

*Author to whom correspondence should be addressed:

José María De Teresa (deteresa@unizar.es)

Instituto de Ciencia de Materiales de Aragón (ICMA)

Universidad de Zaragoza-CSIC

Facultad de Ciencias

Pedro Cerbuna 12. 50009 Zaragoza, Spain.

Tel: +34 876553354

Fax: +34 976761229 


\begin{abstract}
Focused Electron Beam Induced Deposition is a consolidated technique for the growth of three-dimensional (3D) nanostructures. However, this single-step nanofabrication method requires further efforts to optimize simultaneously dimensional and compositional properties, in particular for deposits with a high aspect ratio. More specifically, ferromagnetic 3D nanowires (NWs) with diameters in the sub-50 nm regime and high metallic contents up to 95 at. \% attract great interest to improve the final performance of magnetic nanodevices such as magnetic tips for scanning probe microscopy. In this work, we report on real-time monitoring during chemical purification and structural crystallization processes of ultra-narrow 3D Fe NWs $(<50 \mathrm{~nm}$ in diameter achieved) by post-growth in situ annealing in a transmission electron microscope. NW heating up to $700{ }^{\circ} \mathrm{C}$ in very high vacuum reveals the local increase of the metallic content along the entire NW length concomitant with the growth of large Fe single crystals from initially amorphous compounds. A metallic purity of 95 at. $\%$ is observed in several regions, dramatically boosting the initial $\mathrm{Fe}$ content of 40 at. \%. The real-time in situ tracking of 3D nanostructures during thermal annealing is a key element to design and optimize novel purification processes for the fabrication of customized components to be integrated in spintronic, logic and sensing devices.
\end{abstract}

\footnotetext{
Keywords: focused electron beam induced deposition; iron nanowires; threedimensional nanostructures; composition purification; in-situ transmission electron microscopy
} 


\section{Introduction}

The increasing demand for high-density and low-power data storage, combined with technology approaching quantum limits for downscaling, naturally entails the expansion from two-dimensional (2D) to three-dimensional (3D) architectures to reach ultrahigh density on information storage media. In the case of magnetism-based technologies, magnetic nanowires are the building blocks in the development of future 3D magnetic data storage devices [1]. The functionality of these nanostructures relies on the precise control of domain wall motions with spin polarized currents or magnetic fields in vertical nanowires, to boost the performance of these memories in terms of areal density and power consumption [2]. Focused Electron Beam Induced Deposition (FEBID) is a matured nanofabrication technique, which enables highly localized synthesis of functional nanostructures on almost any material and surface morphology. This directwriting nanofabrication method has an unique status for the development of 2D [3] and 3D [4] objects with spatial resolution in the range of nanometers, without the need of masks, resists, etching- or lift-off processes [5]. The decomposition of a precursor gas adsorbed on the substrate surface by an accurately-directed electron beam enables the deposition of metallic [6], magnetic [7], insulating [8] and superconducting [9] materials only in the areas scanned by the beam. This outstanding versatility yields a broad spectrum of applications, covering circuit edit and mask repair [10], micro- and nanocontacting [11], photodetection [12], nano-sensing [13], magnetomechanical actuation systems [14], plasmonics [15], etc. However, the proper operation of such devices can be compromised when the functional properties of the nanostructures are not ideally tuned. One of FEBID main issues is the existence of chemical impurities due to incompletelydissociated precursor molecules leading to incorporation of unwanted fragments inside the deposit, often containing carbon and oxygen. Purity improvement can be achieved by 
precise control of primary electron beam parameters (beam energy and current), vacuum chamber base pressure, precursor gas flux, substrate temperature, tilt angle of the gas injection system, distance between the nozzle and the substrate, etc [16]. However, even after optimizing these growth parameters, the purity of as-deposited structures often remains moderate [17]. Thus, several strategies have been developed to enhance the deposit purity: in situ [18] and ex situ [19][20] post-annealing treatments at high vacuum and under controlled gas atmosphere [21], use of substrates at high temperatures during growth [22][23], electron beam irradiation of the deposits [24][25], application of high current densities [26] or use of carbon-free precursor gases [27] are the most widely-used. Nearly all these approaches were performed for 2D deposits [28], whereas purification of 3D objects have been scarcely carried out [15][29]. Purification of 3D deposits present specific problems: for instance, the architecture stability due the high-volume shrinkage during heating treatments is critical [30].

FEBID of Fe [31], $\mathrm{Co}$ [32] and $\mathrm{Ni}$ [33] precursors gives rise to magnetic deposits in which low metallic content leads to degraded magnetic properties. High purity $2 \mathrm{D} \mathrm{Fe}$ and $\mathrm{Co}$ deposits ( $\sim 95$ at. $\%)$ can be grown after optimizing the nanofabrication parameters [34][35]. Nevertheless, in the case of 3D nanostructures with a high aspect ratio, as relevant for vertical nanowires (NWs), the metallic content is drastically reduced for decreasing diameter. Moreover, the natural oxidation of the surface upon air exposure (typically $5 \mathrm{~nm}$ thick) becomes critical as the surface-to-volume ratio significantly increases and impairs the ferromagnetic properties of the nanostructures due to the formation of non-ferromagnetic species [36][37]. Therefore, novel strategies such as onpurpose core-shell designs [38] or ex situ annealing treatments in high vacuum [29] have been pursued to prevent and solve these issues. 
While most annealing studies have been performed in post-process conditions (e.g. after each temperature step), real-time evaluation concerning chemical composition, overall morphology and local crystallinity is still rare, even though such studies would provide deeper insight in their fundamental nature. Based on this motivation, we here use in situ Transmission Electron Microscopy (TEM) for studying the implications of thermal postgrowth annealing on Fe based FEBID NWs with atomic resolution [39]. The possibility to analyze the results in real-time at each temperature step allows getting an insight on the process dynamics, confining the required purification time scales. In more detail, we focus on the formation and distribution of highly-pure metallic Fe phases, including their crystallinity in real time (sample A). These results are compared with the ex situ annealing experiments performed on specimens that initially showed a higher metal content (samples B-F) (see Supporting Information). The two setups used for the growth in different laboratories (Austria and Spain) led to different chemical composition of the asgrown nanowires, allowing us to investigate the behavior of thermal post-processing of 3D magnetic NWs with a broader view. This improved understanding is the basis for further optimization of magnetic NWs for future applications in sensing, memory and logic.

\section{Experimental}

2.1 Growth of 3D Fe NWs by FEBID. Although FEBID 3D-nanoprinting allows the fabrication of complex 3D designs [16], this study representatively uses a pillar geometry for simplicity. The nanostructures (sample A; Austrian laboratory) were fabricated in a commercial FEI Nova 200 dual beam microscope equipped with a thermal field emission gun (FEG) electron column and a standard gas injection system (GIS) for depositing Fe using $\mathrm{Fe}_{2}(\mathrm{CO})_{9}$ precursor gas. The NWs were grown at room temperature on a heating chip (Wildfire S3, DENSsolutions, The Netherlands) prepared to be loaded into a 
Wildfire TEM holder for subsequent in situ heating experiments.
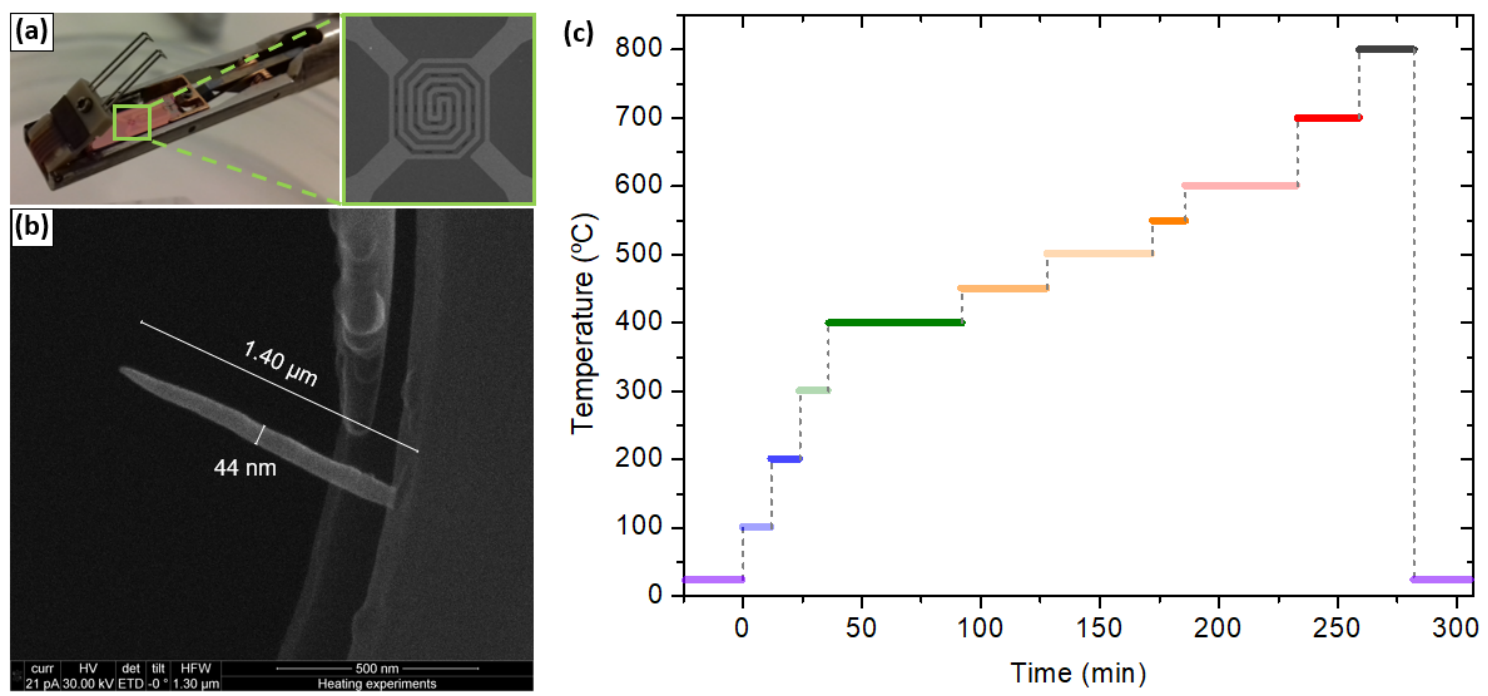

Figure 1. (a) Heating chip mounted in the TEM holder with (b) an Fe-FEBID nanowire grown for real-time TEM annealing experiments. (c) Temperature profiles for the FeFEBID nanowire studied.

The chip substrate includes oval holes where the suspended nanostructures can be imaged, as shown in Figure 1(a-b). The chip was tilted during the vertical growth of the NW, so that the nanostructure projection falls into the hole once the chip recovers the horizontal position. After the optimization of the growth parameters, NWs of very small diameter $(<50 \mathrm{~nm})$, with a high aspect ratio $(>30)$ and an initial moderately-low Fe relative composition ( $\sim 40$ at. $\%)$ were obtained. All deposits were fabricated using an electron beam voltage of $30 \mathrm{kV}$, electron beam current of $21 \mathrm{pA}$, growth pressure of $4 \times 10^{-7} \mathrm{mbar}$ (working pressure minus base pressure), precursor temperature of $\sim 30{ }^{\circ} \mathrm{C}$ and a single spot exposure for 600 seconds.

2.2 Post-growth annealing in the Transmission Electron Microscope. The postgrowth annealing in high vacuum was performed in situ in an FEI Titan Cube G2 60-300 using a Wildfire TEM holder from DENSsolutions (sample A). Figure 1(c) shows the temperature profile during the experiment, where individual annealing times are colorcoded: $100{ }^{\circ} \mathrm{C}(12 \mathrm{~min}), 200{ }^{\circ} \mathrm{C}(12 \mathrm{~min}), 30{ }^{\circ} \mathrm{C}(12 \mathrm{~min}), 400{ }^{\circ} \mathrm{C}(56 \mathrm{~min}), 450{ }^{\circ} \mathrm{C}(36$ 
$\min ), 500{ }^{\circ} \mathrm{C}(44 \mathrm{~min}), 550{ }^{\circ} \mathrm{C}(14 \mathrm{~min}), 600^{\circ} \mathrm{C}(47 \mathrm{~min}), 700{ }^{\circ} \mathrm{C}(26 \mathrm{~min})$ and $800{ }^{\circ} \mathrm{C}(23$ $\min )$.

\subsection{In situ Transmission Electron Microscopy characterization. Scanning} Transmission Electron Microscopy (STEM) imaging in combination with Electron Energy Loss Spectroscopy (EELS) was performed using an FEI Titan Cube G2 60-300 equipment operated at $300 \mathrm{kV}$ and fitted with a high brightness electron gun (X-FEG) with monochromator and a $\mathrm{C}_{\mathrm{S}}$ probe corrector $(\mathrm{DCOR})$, which produces an electron probe below $0.7 \AA$ in STEM imaging, and a Gatan Imaging Filter (GIF) Quantum. The STEMEELS experiments were carried out with a 19.7 and 20.5 mrad convergence and collection semiangles, respectively, an energy dispersion of $0.5 \mathrm{eV} /$ pixel with a resolution of $1.5 \mathrm{eV}$ (FWHM of the zero-loss peak), a GIF aperture of $5 \mathrm{~mm}$, a camera length of $58 \mathrm{~mm}$, a pixel time of $10 \mathrm{~ms}$ and an estimated beam current of $160 \mathrm{pA}$. Annular Dark Field (ADF) images were acquired with a Gatan ADF detector in the GIF with an inner detector angle of $38 \mathrm{mrad}$ and an outer detector angle of $137 \mathrm{mrad}$.

\section{Results}

The nanofabrication of 3D Fe NWs with $\sim 40$ at. $\%$ metallic content and $\sim 50 \mathrm{~nm}$ in diameter was performed via FEBID. A live monitoring of the features during the annealing allowed the in situ characterization of the structural and compositional variations from $24{ }^{\circ} \mathrm{C}$ to $800{ }^{\circ} \mathrm{C}$. This gave us the opportunity of choosing different annealing times at each temperature depending on the changes observed or required. Furthermore, the nanostructures can be tailored until the desired properties are achieved. Based on this consideration, the sample was kept at certain temperatures as long as significant variations were detected. Figure 2 shows a schematic illustration of the dimensional and organizational modifications of the components depending on the annealing temperature as obtained in the experiments. In brief, purified $(\sim 95$ at. $\% \mathrm{Fe})$ 
crystalline regions of the NW were obtained, reducing the lateral resolution down to $\sim 30$ $\mathrm{nm}$ in specific areas, but retaining the general shape of the nanostructure.

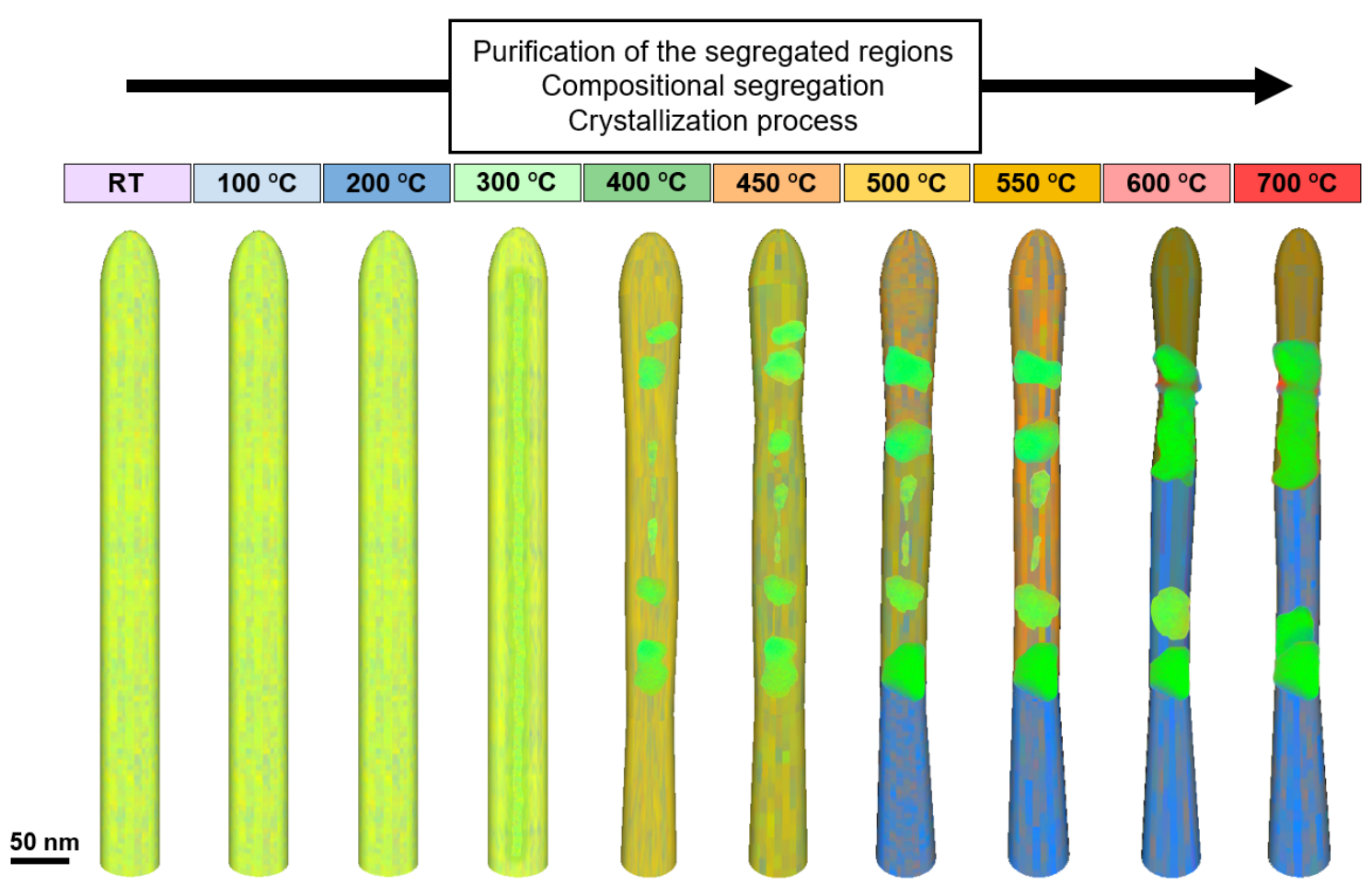

Figure 2. Sketch of the morphology, dimensions and composition distribution of an FeFEBID NW as a function of the annealing temperature as observed in the experiments. $\mathrm{Fe}, \mathrm{O}$ and $\mathrm{C}$ are depicted in green, red and blue respectively.

ADF imaging and EELS compositional mapping in STEM mode have been used to trace the morphological, structural and chemical evolution of the NW with increasing annealing temperatures. Figure 3 illustrates the general features observed upon heating by focusing the attention on a central region of the NW. The average diameter tends to decrease as the temperature value raises, obtaining a $40 \%$ reduction of the thickness in certain regions, dropping down from $\sim 50 \mathrm{~nm}$ at $24{ }^{\circ} \mathrm{C}$ to $\sim 30 \mathrm{~nm}$ at $700{ }^{\circ} \mathrm{C}$. This is due to an expected volume shrinkage caused by the progressive reduction of carbon and oxygen content, due to the formation of volatile $\mathrm{CO}_{\mathrm{x}}$ compounds as previously discussed [25][40]. Chemical analyses reveal that the NW core contains $\sim 40$ at. $\%$ Fe once they are exposed to air, before the annealing treatment. As shown in greater detail in Figure 4, 
regarding the crystalline texture, the as-deposited NW has a nanocrystalline structure which begins to change in the early stages of the experiment.

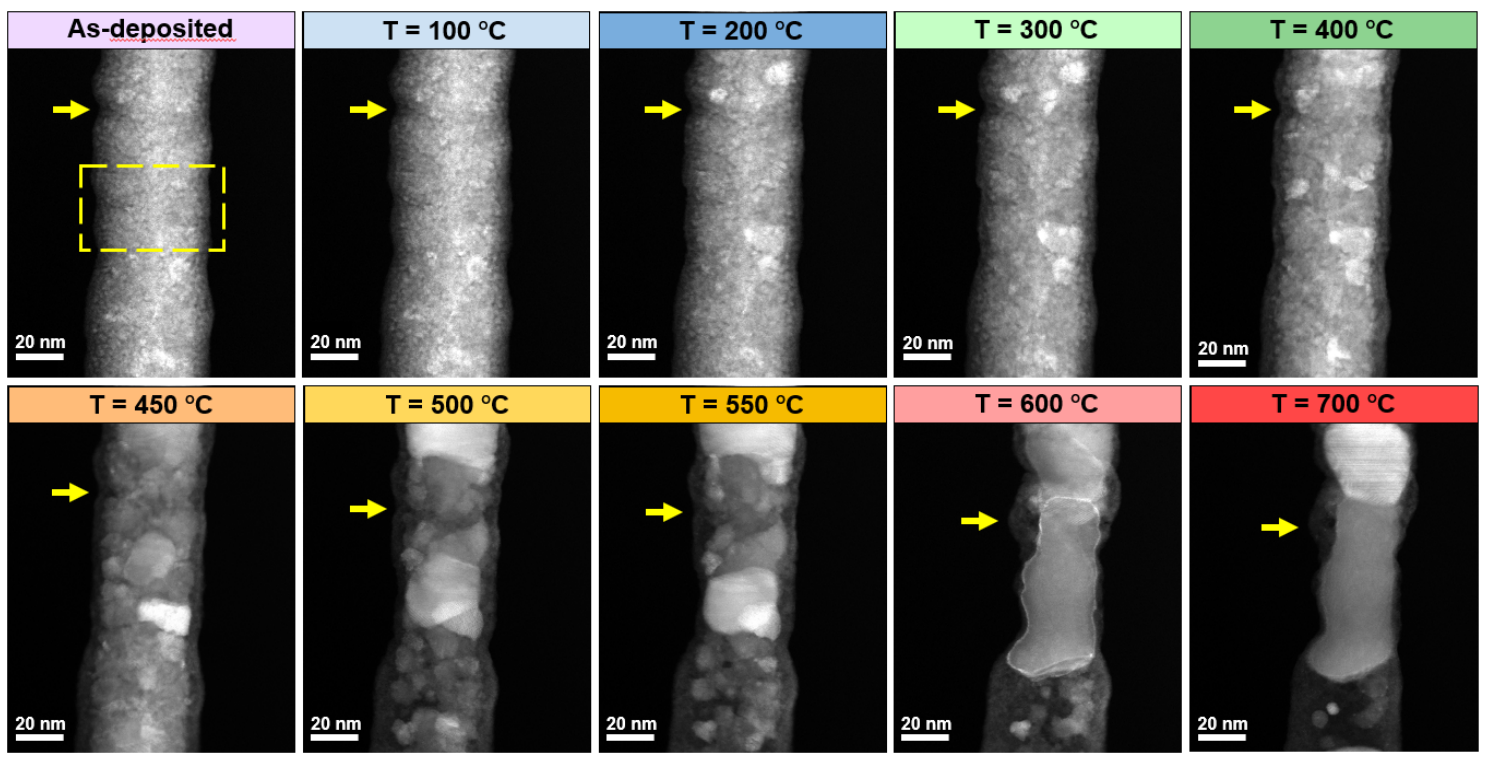

Figure 3. ADF-STEM images of the central section of an as-deposited Fe-FEBID nanowire, sequentially annealed at 100, 200, 300, 400, 450, 500, 550, 600 and $700{ }^{\circ} \mathrm{C}$. Yellow arrows are guides to the eye indicating the same point of the nanowire. The yellow dashed square in the image of the as-deposited nanowire indicates the area highlighted in Figure 4.
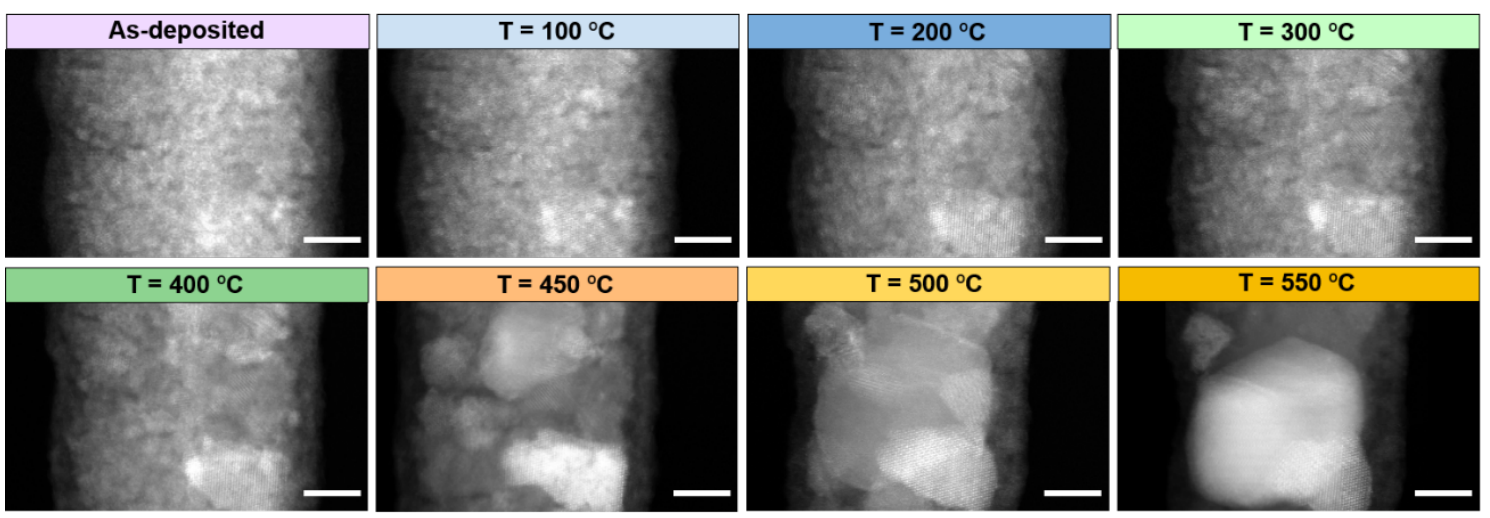

Figure 4. High-resolution ADF-STEM images of the area indicated with yellow dashed square in Figure 3 of an as-deposited Fe-FEBID nanowire, sequentially annealed at 100, $200,300,400,450,500$ and $550^{\circ} \mathrm{C}$. Scale bars are $10 \mathrm{~nm}$ in all images.

From $24{ }^{\circ} \mathrm{C}$ to $300{ }^{\circ} \mathrm{C}$, only minor structural changes are observed. At $100{ }^{\circ} \mathrm{C}$ crystallites up to $\sim 10 \mathrm{~nm}$ begin to grow in specific regions, suggesting that a global homogeneous composition in the core is kept with the Fe starting to segregate from the rest of the components on specific areas. The low electron current density $\left(<40 \mathrm{nA} / \mathrm{nm}^{2}\right)$ concentrated on the nanowire ensures that the driving force of the crystal emergence is 
provided by the heater temperature rise rather than by the irradiation-enhanced diffusion phenomenon [41][42]. When the sample is heated to $200{ }^{\circ} \mathrm{C}$, new isolated ordered arrangements of atoms turn up, and oriented in different directions at several areas, whilst maintaining the general structural shape of the NW. At $300^{\circ} \mathrm{C}$ the number of high-purity Fe nanocrystals increases inside the core, giving rise to an overlap amongst them. In addition, general compositional changes happen, noticing a richer Fe central trace found along the long axis of the nanostructure (see Figure 5). Raising the temperature up to 400 ${ }^{\circ} \mathrm{C}$ favours the clustering of the crystals, giving rise to an inhomogeneous distribution of Fe, which produces areas with remarkable higher metallic content. Indeed, a particularly active redistribution of the $\mathrm{Fe}, \mathrm{O}$ and $\mathrm{C}$ can be noticed as a function of time, which is illustrated in Figures 5. At $450^{\circ} \mathrm{C}$ a grain size growth takes place with crystals spreading all over the core thickness in certain regions. 


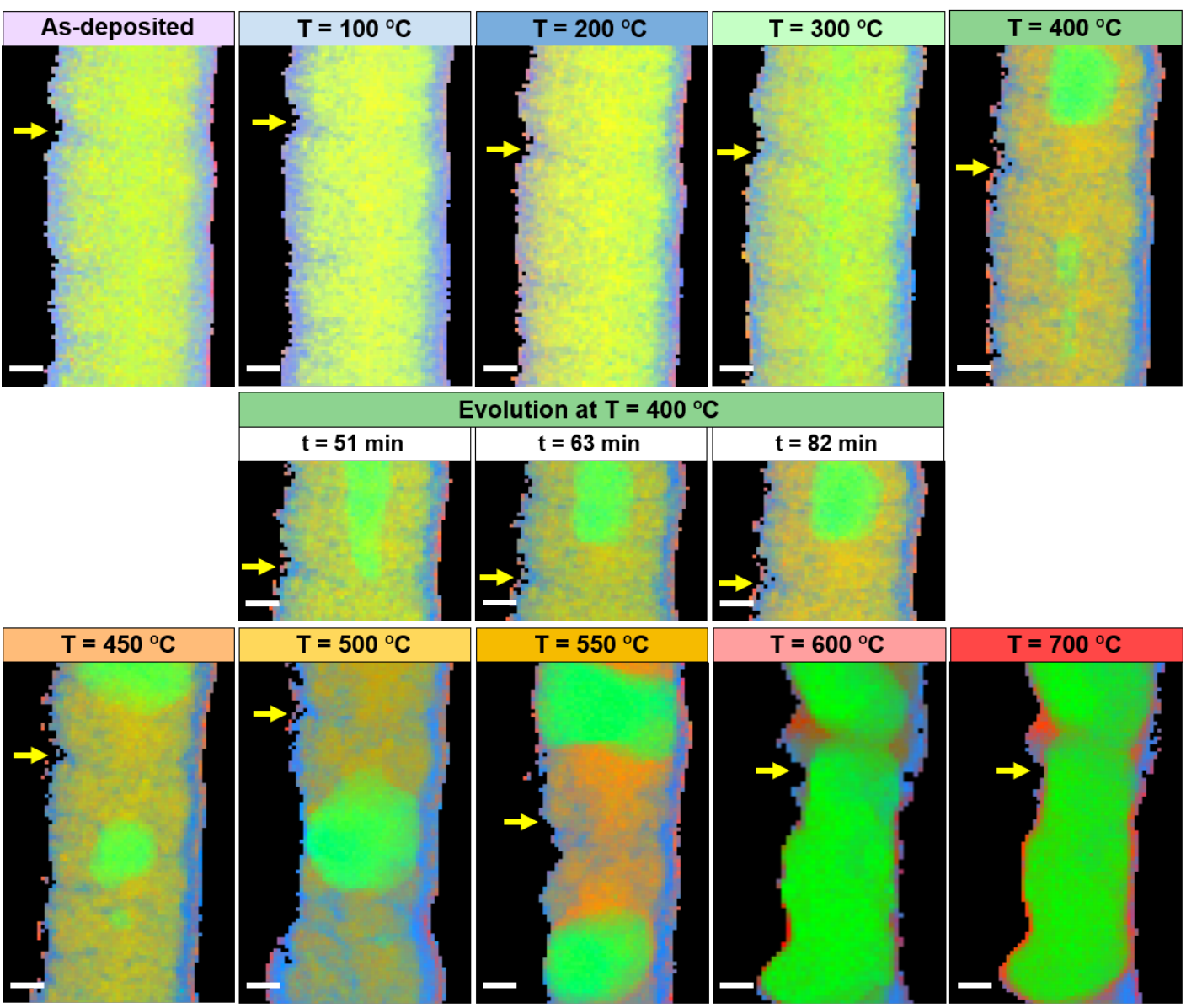

Figure 5. STEM-EELS chemical maps of the central section of an as-deposited FeFEBID nanowire, sequentially annealed at 100, 200, 300, 400, 450, 500, 550, 600 and $700{ }^{\circ} \mathrm{C}$, showing the spatial distribution of $\mathrm{Fe}, \mathrm{O}$, and $\mathrm{C}$ in green, red and blue, respectively. Yellow arrows are guides to the eye indicating the same point of the nanowire. Scale bars are $10 \mathrm{~nm}$ in all images.

Figure 4 shows a clear change in the NW microstructure, virtually removing most of the remained amorphous material in Fe-rich parts. It is also noteworthy that, since Fe-rich areas appear inside the NW, therefore C-rich and O-rich areas should appear in other areas. Moreover, given that the initial Fe content was low ( 40 at. \%), the complete purification throughout the whole nanostructure is not expected to occur whilst keeping the total volume constant, and similar behaviour was found in the 3D samples B-F with higher initial metallic content ( $\geq 75$ at. \%) (see Supporting Information). At $500{ }^{\circ} \mathrm{C}$ the diameter is not uniform anymore along the length of the nanostructure, considerably reducing its value below $45 \mathrm{~nm}$ in some sections. As illustrated in Figure 5, large Fe 
grains grow in size, at the expense of surrounding smaller grains, to reach lateral sizes close to the $\mathrm{NW}$ width. At $550^{\circ} \mathrm{C}$, the recrystallization of the Fe-rich regions is completed and has produced the expected large crystals of $\alpha$-Fe (bcc). This correlates very well with the result obtained from samples B-F (see Supporting Information), and with the previous observation of the transformation of amorphous $\mathrm{Fe}: \mathrm{C}: \mathrm{O}$ as-grown nanocomposites into $\alpha$-Fe single-crystals around this temperature [43]. In addition to that, when the component distribution does not change anymore as a function of time at $550{ }^{\circ} \mathrm{C}$, a temperature increase to $600{ }^{\circ} \mathrm{C}$ is required to stimulate further compositional dynamics. Here, Fe crystal migration in some areas of the NW is powerfully promoted, with the grain front advancing with a mean speed of $\sim 26 \mathrm{pm} / \mathrm{s}$ (see Video S1). For the sake of comparison, iron carbon alloy nanoparticles were found to move inside a $\mathrm{C}$ nanopillar at $8 \mathrm{~nm} / \mathrm{s}$ under comparable temperatures [44]. In the case of 3D Fe-FEBID NWs, the diffusion suggests being subject to the specific morphology of the regions, the available metallic sources around the active grain front when they are still present and the possible crystal accommodation within the NW. The NW segment represented in Figure 5 shows a remarkable expansion of the Fe content along that section. However, despite not displaying the whole length of the nanostructure to have a better assessment of the fine compositional details, it is important to highlight that this local purification leads to the accumulation of $\mathrm{C}$ and $\mathrm{O}$ in other regions of the NW as illustrated in Figures 2 and 3. At $700{ }^{\circ} \mathrm{C}$, the NW reveals a final diameter of approximately $30 \mathrm{~nm}$ in the thinnest regions. At this point, it is likely that the growth of large $\alpha$-Fe crystals is halted as the supply of small iron particles at close distances has stopped. The adopted configuration resembles the one obtained for the previous temperature stage and it can be assumed that the segregation between the components is finished. At $800^{\circ} \mathrm{C}$, the NW suddenly bends at the upper part, suffering an important deformation and therefore losing the $3 \mathrm{D}$ pillar 
original shape (see Figure S1). Hence, the large amount of C initially contained inside the structure not only hampers obtaining a homogeneously purified structure, but also leads to the collapse of the NW structure at high annealing temperatures. Besides, some Fe clusters are observed moving around inside the more carbonaceous core areas whereas the $\mathrm{O}$ almost disappears.

To obtain quantitative information about the $\mathrm{Fe}, \mathrm{O}$ and $\mathrm{C}$ relative compositions, STEMEELS profiles were performed along the length (Figure 6) and the diameter (Figure 7) of the NW at each annealing temperature. For the first one, the profiles were acquired approximately in the same areas depicted in Figures 3 and 5 and by integrating over the central $\sim 12 \mathrm{~nm}$. For the second one, the plots over the thickness of the nanostructure does not provide fair information about the overall composition but allow studying the shell composition and obtaining numerical values of the relative contents of $\mathrm{Fe}, \mathrm{O}$ and $\mathrm{C}$ in specific sections. In this case, since there is an active displacement of the components depending on the temperature, we have selected the regions which illustrate the general behaviour of the composition at the shell and the highest Fe percentage found throughout the NW for each annealing temperature. Figure 6 indicates that the initial $\sim 40$ at. $\% \mathrm{Fe}$, $\sim 40$ at. $\% \mathrm{O}$ and $\sim 20$ at. $\% \mathrm{C}$ is homogeneously distributed in the core from $24^{\circ} \mathrm{C}$ to 300 ${ }^{\circ} \mathrm{C}$. However, this calculation considers the contribution coming from the shell because the whole thickness of the NW is integrated in the STEM-EELS data collection. As shown in Figure 7(a-d), in this temperature range, a $\sim 7 \mathrm{~nm}$ shell composed by $\sim 10$ at. $\% \mathrm{Fe}, \sim 30$ at. $\% \mathrm{O}$ and $\sim 60$ at. $\% \mathrm{C}$ represents nearly the $30 \%$ of the total width. Thus, one can assume that the real metallic content inside the core is much higher than the one displayed for the total volume. Another relevant fact is the absence of the standard $\sim 5 \mathrm{~nm}$ surface oxidation layer which typically covers magnetic FEBID nanostructures with higher metallic content [45]. This suggests the instantaneous formation of a $\mathrm{C}$ shell during the 
growth of the original Fe NW, avoiding the natural oxidation process of the metal species once the nanostructure is removed from the nanofabrication vacuum chamber. As a result, the $\mathrm{O}$ signal decreases from the core to the shell. At $400{ }^{\circ} \mathrm{C}$ and $450{ }^{\circ} \mathrm{C}$, a strong difference in the Fe contents along the length has been detected. According to Figure 6(e-f), the regions where a cluster of Fe appears improve the metallic composition up to the range between 50-60 at. \% at the maximum, as well as the $\mathrm{O}$ signal decreases down to $\sim 20$ at. $\%$. These data correlate appropriately with Figure $7(\mathrm{e}-\mathrm{f})$. At $500{ }^{\circ} \mathrm{C}$, the Fe relative composition exceeds 60 at. $\%$ in the areas with the highest metallic content, where the $\mathrm{O}$ is reduced below 20 at. $\%$. At $550{ }^{\circ} \mathrm{C}$, the Fe content in those regions increases to around 70 at. $\%$. Simultaneously, the $\mathrm{C}$ content starts to decrease at the shell and percentages below 50 at. $\%$ are obtained for the first time. At the same time, the $\mathrm{O}$ signal decreases drastically in central regions. At $600{ }^{\circ} \mathrm{C}$, looking at the transversal profile analysis shown in Figure 7(i), the C signal varies the tendency and decreases dramatically down to zero value in some sections. Consequently, the Fe is expanded all over the NW thickness, the recrystallization and the local purification is accomplished locally, approaching almost $\sim 95$ at. $\%$ with the $\mathrm{O}$ layer present at the shell. 

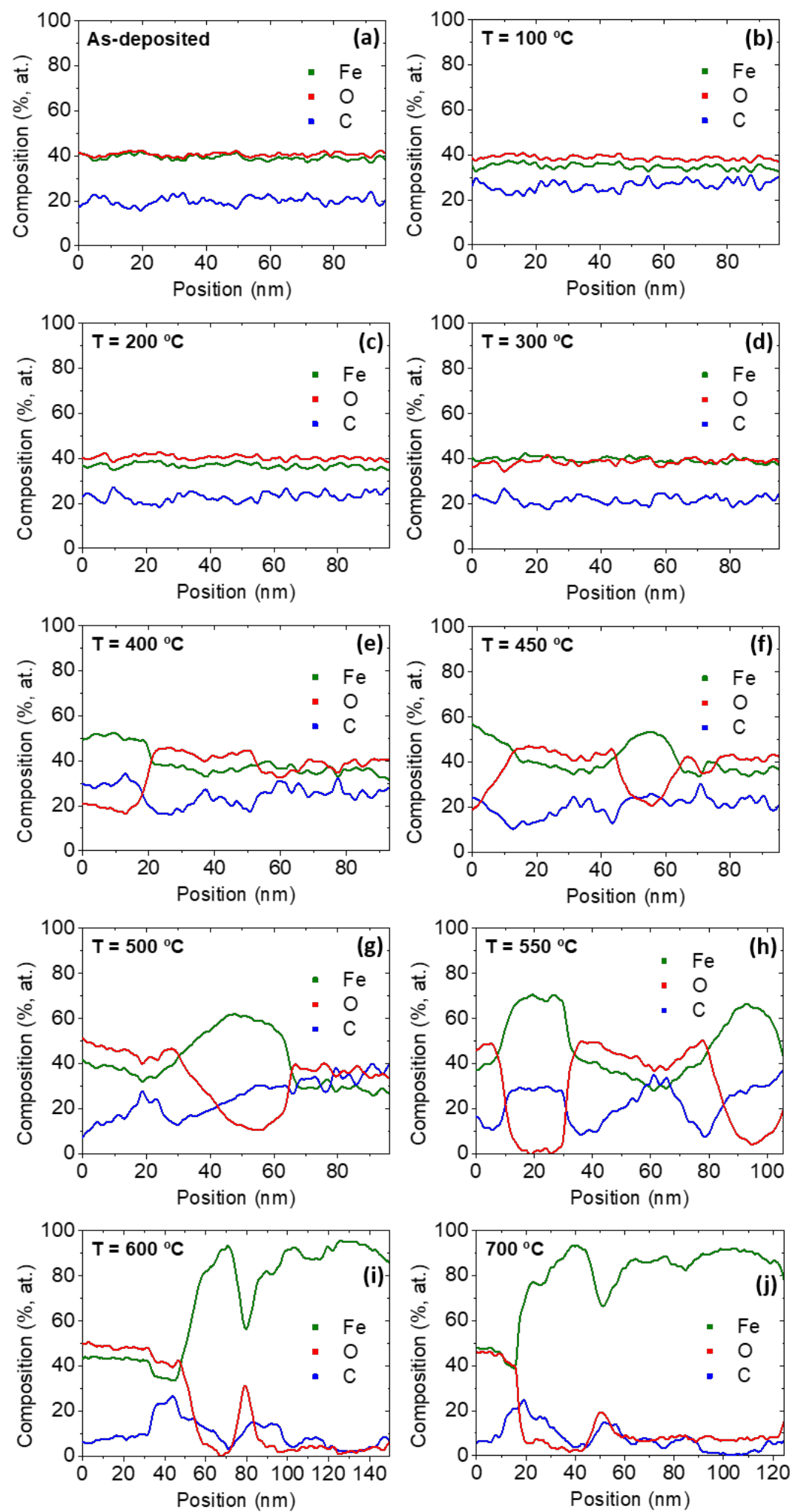

Figure 6. STEM-EELS profiles as a function of the length of (a) an as-deposited FeFEBID nanowire and the same nanostructure sequentially annealed at (b) 100, (c) 200, (d) 300, (e) 400, (f) 450, (g) 500, (h) 550, (i) 600 and (j) $700{ }^{\circ} \mathrm{C}$. The Fe, O and $\mathrm{C}$ compositions are represented in green, red and blue respectively. 

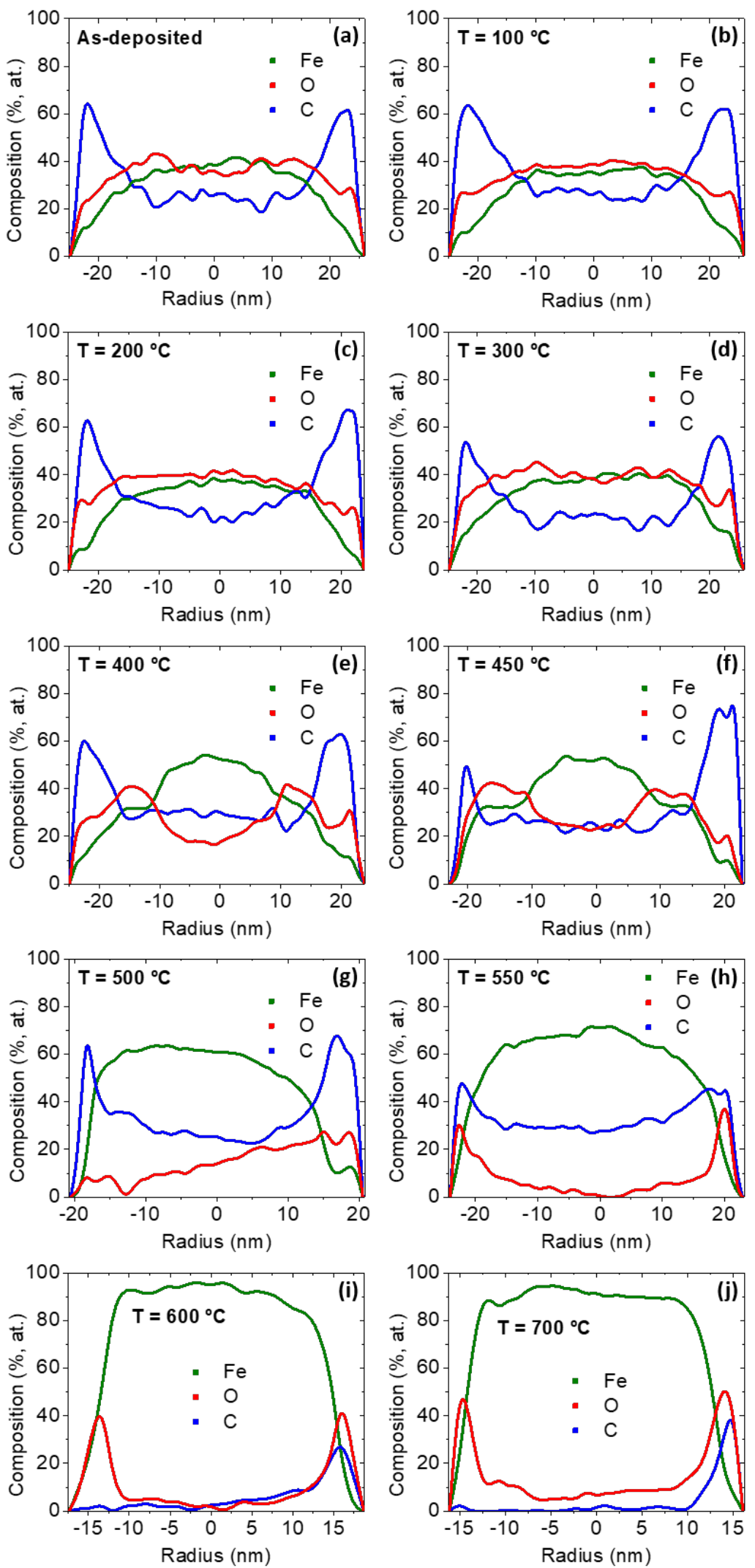

Figure 7. STEM-EELS profiles of the relative composition of the highest-metallic areas as a function of the radius for (a) an as-deposited Fe-FEBID NW and the same nanostructure annealed at (b) 100, (c) 200, (d) 300, (e) 400, (f) 450, (g) 500, (h) 550, (i) 600 and (j) $700{ }^{\circ} \mathrm{C}$. 
Also, the Fe signal generally coincides with the $\mathrm{O}$ one in the external areas, which demonstrates that metallic material is eventually oxidized. Adding this to the overall sharp reduction of the diameter, the ferromagnetic diameter shrinks to $\sim 25 \mathrm{~nm}$. Finally, at $700{ }^{\circ} \mathrm{C}$ no significant changes can be noted with respect to the previous temperature, and further heating is not expected to bring any major improvements on the purity.

\section{Discussion}

In order to evaluate the degree of Fe purification with temperature, Figure 8(a) displays the radial dependence of the average Fe composition found in the highest-metallic area of the NW at each annealing temperature. These higher purification sections may vary their location from one temperature to another. A clear increase of the highest Fe content as a function of the temperature can be appreciated in Figure 8(b). Simultaneously, the diameter evolution in these regions follows the opposite trend, decreasing down to $33 \mathrm{~nm}$, attaining a remarkable lateral resolution. Thus, sections of virtually pure Fe can be obtained by means of this annealing treatment from a nanowire with low initial $\mathrm{Fe}$ content, but the full metal purification along the nanowire is not achieved. The obtained nanoscale phase segregation will entail non-homogeneous physical properties in the nanowire, which will have a significant impact on the performance of nanodevices based on this material. As shown in the Supplementary File, in Fe FEBID nanowires with higher initial Fe content, the annealing process also promotes the phase segregation in areas with higher and with lower Fe content. We can compare this behaviour with similar annealing processes conducted in Co 3D NWs grown by FEBID using the $\mathrm{Co}_{2}(\mathrm{CO})_{8}$ precursor, which showed a complete purification and recrystallization of the nanowires at $600{ }^{\circ} \mathrm{C}$ [46]. This indicates that a different mechanism is governing the purification process in both nanomaterials, with profound implications on their functionality. 

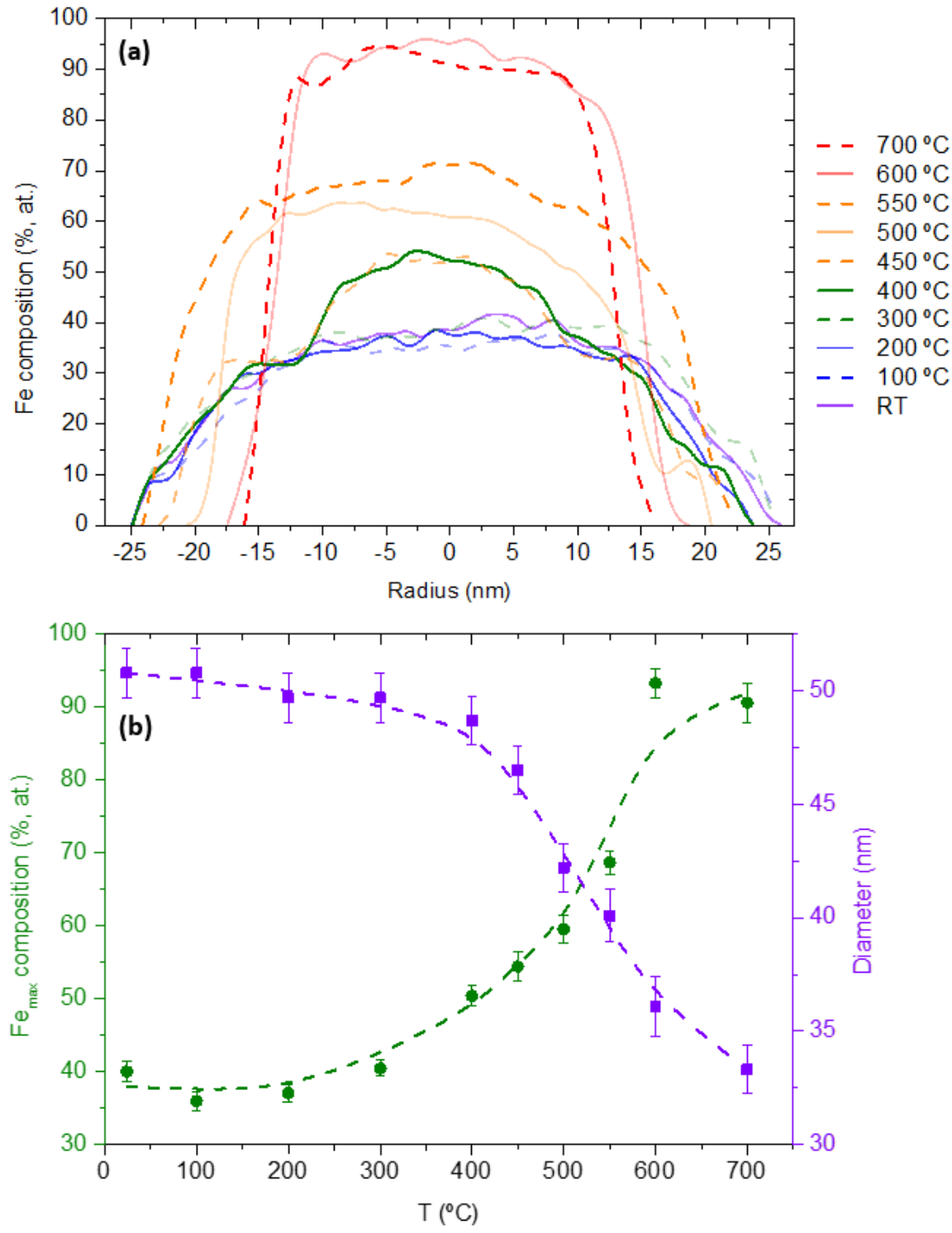

Figure 8. (a) STEM-EELS profiles of the average Fe relative composition as a function of the radius in the areas with the highest metallic content of the Fe-FEBID nanowire found at each temperature. (b) Maximum Fe relative composition (circle symbol) and average total diameter (square symbol) in the selected NW area shown in Figure 4 as a function of the annealing temperature.

In the case of applications requiring magnetic continuity, such as in magnetic domainwall conduits [1], annealed Fe FEBID nanowires would not be appropriate. However, in the case of magnetic sensing devices such as Hall sensors, the use of a nanomaterial with purified magnetic areas surrounded by non-magnetic areas could be beneficial [47]. Such annealed Fe FEBID nanowires may also be of interest to magnetic tips used for Magnetic Force Microscopy for two reasons: the enhancement of the spatial resolution due to the 
reduction of the nanowire diameter and the diminishment of their magnetic invasiveness, currently needed in the measurement of magnetic skyrmions [48]. Another potential application of annealed Fe FEBID nanostructures could be in the field of catalysis, given that the small metallic nanoparticles formed could act as catalytical centres for the growth of carbon nanotubes, as shown in the past with Co FEBID nanodots [49].

\section{Conclusions}

In summary, post-growth annealing treatment under high-vacuum conditions of ultrathin 3D ferromagnetic Fe nanowires $(<50 \mathrm{~nm})$ fabricated by FEBID with the $\mathrm{Fe}_{2}(\mathrm{CO})_{9}$ precursor has been carried out. The morphological, compositional and crystallinity changes as a function of the temperature and time have been monitored in real time inside a transmission electron microscope, shedding light on the nanoscale processes involved during the annealing process. According to the obtained results, annealing up to $700{ }^{\circ} \mathrm{C}$ of an as-grown homogeneous nanocrystalline nanowire with an initial metallic content of about 40 at. \% Fe induces the coexistence of Fe-purified and crystallized regions in the nanowire with other Fe-deficient amorphous regions. Thus, instead of the full metal purification, this annealing method provokes a strong phase segregation in the nanowire. Our work underlines the importance of in-situ studies with nanoscale resolution for the optimization of nanomaterials, and the understanding of their functionality not in terms of their average physical properties, but of the physical properties of their individual constituents. Potential applications of these results in magnetic sensing and catalysis have been discussed. 


\section{Acknowledgements}

Authors acknowledge financial support from the Spanish Ministry of Economy and Competitiveness (MINECO) through the projects MAT2017-82970-C2-1-R and MAT2017-82970-C2-2-R, and from the Aragon Regional Government (Construyendo Europa desde Aragón) through project and E13_17R with European Social Fund funding.

J. P.-N. grant is funded by the Ayuda para Contratos Predoctorales para la Formación de Doctores (BOE 12/06/15) of the Spanish MINECO with the participation of the European Social Fund. The financial support by the Austrian Federal Ministry for Digital and Economic Affairs and the National Foundation for Research, Technology and Development is gratefully acknowledged. Financial support was also received from the FFG Austria in the frame of the "Beyond Europe" initiative (Project AIM, Nr. 11056459). This project has received funding from the European Union's Horizon 2020 research and innovation programme under grant agreement No. 823717 - ESTEEM3. Experimental help by R. Krisper from the Institute of Electron Microscopy and Nanoanalysis is acknowledged.

\section{References}

[1] S.S.P. Parkin, M. Hayashi, L. Thomas, Magnetic domain-wall racetrack memory, Science 320, (2008) 190-194.

[2] M. Vázquez, Magnetic nano- and microwires: design, synthesis, properties and applications, first ed., Elsevier, 2015.

[3] E. Nikulina, O. Idigoras, P. Vavassori, A. Chuvilin, A. Berger, Magneto-optical magnetometry of individual $30 \mathrm{~nm}$ cobalt nanowires grown by electron beam induced deposition, Appl. Phys. Lett. 100 (2012) 142401.

[4] A. Fernández-Pacheco, L. Serrano-Ramón, J.M. Michalik, M.R. Ibarra, J.M. De 
Teresa, L. O’Brien, D. Petit, J. Lee, R.P. Cowburn, Three dimensional magnetic nanowires grown by focused electron-beam induced deposition, Sci. Rep. 3 (2013) 1492.

[5] I. Utke, P. Hoffmann, J. Melngailis, Gas-assisted focused electron beam and ion beam processing and fabrication, J. Vac. Sci. Technol. B 26 (2008) 1197-1276.

[6] D.N. Madsen, K. Mølhave, R. Mateiu, A.M. Rasmussen, M. Brorson, C.J.H. Jacobsen, P. Bøggild, Soldering of nanotubes onto microelectrodes, Nano Lett. 3 (2003) 47-49.

[7] G.C. Gazzadi, J.J.L. Mulders, P. Trompenaars, A. Ghirri, A. Rota, M. Affronte, S. Frabboni, Characterization of a new cobalt precursor for focused beam deposition of magnetic nanostructures, Microelectron. Eng. 88 (2011) 19551958.

[8] S. Lipp, L. Frey, C. Lehrer, B. Frank, E. Demm, S. Pauthner, H. Ryssel, Tetramethoxysilane as a precursor for focused ion beam and electron beam assisted insulator $\left(\mathrm{SiO}_{x}\right)$ deposition, J. Vac. Sci. Technol. B 14 (1996) 39203923.

[9] J. Barzola-Quiquia, S. Dusari, C. Chiliotte, P. Esquinazi, Andreev reflection and granular superconductivity features observed in mesoscopic samples using amorphous tungsten carbide superconductors, J. Supercond. Nov. Magn. 24 (2011) 463-469.

[10] T. Liang, E. Frendberg, B. Lieberman, A. Stivers, Advanced photolithographic mask repair using electron beams, J. Vac. Sci. Technol. B 23 (2005) 3101-3105.

[11] N. Sharma, P. Vugts, C. Daniels, W. Keuning, J.T. Kohlhepp, O. Kurnosikov, B. 
Koopmans, Multi-channel Andreev reflection in Co-W nanocontacts fabricated using focused electron/ion beam induced deposition, Nanotechnology, 25 (2014) 495201.

[12] K. Makise, K. Mitsuishi, M. Shimojo, K. Furuya, A nanosized photodetector fabricated by electron-beam-induced deposition, Nanotechnology 20 (2009) 2-6.

[13] M. Moczała, K. Kwoka, T. Piasecki, P. Kunicki, A. Sierakowski, T. Gotszalk, Fabrication and characterization of micromechanical bridges with strain sensors deposited using focused electron beam induced technology, Microelectron. Eng. $176(2017) 111-115$.

[14] P. Vavassori, M. Pancaldi, M. J. Perez-Roldan, A. Chuvilin, A. Berger, Remote magnetomechanical nanoactuation, Small 12 (2016) 1013-1023.

[15] R. Winkler, F.-P. Schmidt, U. Haselmann, J.D. Fowlkes, B.B. Lewis, G. Kothleitner, P.D. Rack, H. Plank, Direct-write 3D nanoprinting of plasmonic structures, ACS Appl. Mater. Interfaces 9 (2017) 8233-8240.

[16] R. Winkler, B.B. Lewis, J.D. Fowlkes, H. Plank, P.D. Rack, High-fidelity 3Dnanoprinting via focused electron beams: growth fundamentals, ACS Appl. Nano Mater. 1 (2018) 1014-1027.

[17] T. Bret, I. Utke, A. Bachmann, and P. Hoffmann, In situ control of the focusedelectron-beam-induced deposition process, Appl. Phys. Lett. 83 (2003) 40054007.

[18] M.V. Puydinger Dos Santos, M.F. Velo, R.D. Domingos, Y. Zhang, X. Maeder, C. Guerra-Nuñez, J.P. Best, F.Béron, K.R. Pirota, S. Moshkalev, J.A. Diniz, I. Utke, Annealing-based electrical tuning of cobalt-carbon deposits grown by 
focused-electron-beam-induced deposition, ACS Appl. Mater. Interfaces 8 (2016) 32496-32503.

[19] K. Mitsuishi, M. Shimojo, M. Tanaka, M. Takeguchi, K. Furuya, Resolution in new nanofabrication technique combining electron-beam-induced deposition and low-energy ion milling, Jpn. J. Appl. Phys. 44 (2005) 5627-5630.

[20] M. Shimojo, M. Takeguchi, M. Tanaka, K. Mitsuishi, K. Furuya, Electron beaminduced deposition using iron carbonyl and the effects of heat treatment on nanostructure, Appl. Phys. A 79 (2004), 1869-1872.

[21] D. Belić, M.M. Shawrav, M. Gavagnin, M. Stöger-Pollach, H.D. Wanzenboeck, E. Bertagnolli, Direct-write deposition and focused-electron-beam-induced purification of gold nanostructures, ACS Appl. Mater. Interfaces 7 (2015) 24672479.

[22] R. Córdoba, J. Sesé, J.M. De Teresa, M.R. Ibarra, Microelectronic engineering high-purity cobalt nanostructures grown by focused-electron-beam-induced deposition at low current, Microelectron. Eng. 87 (2010) 1550-1553.

[23] J.J.L. Mulders, L.M. Belova, A. Riazanova, Electron beam induced deposition at elevated temperatures: compositional changes and purity improvement, Nanotechnology 22 (2011) 055302.

[24] H. Plank, J.H. Noh, J.D. Fowlkes, K. Lester, B.B. Lewis, and P.D. Rack, Electron-beam-assisted oxygen purification at low temperatures for electronbeam-induced Pt deposits: towards pure and high-fidelity nanostructures, ACS Appl. Mater. Interfaces 6 (2014) 1018-1024.

[25] B. Geier, C. Gspan, R. Winkler, R. Schmied, J.D. Fowlkes, H. Fitzek, S. Rauch, 
J. Rattenberger, P.D. Rack, H. Plank, Rapid and highly compact purification for focused electron beam induced deposits: a low temperature approach using electron stimulated $\mathrm{H}_{2} \mathrm{O}$ reactions, J. Phys. Chem. C 118 (2014) 14009-14016.

[26] G.C. Gazzadi, S. Frabboni, Structural transitions in electron beam deposited Cocarbonyl suspended nanowires at high electrical current densities, Beilstein J. Nanotechnol. 6 (2015) 1298-1305.

[27] K.L. Klein, S.J. Randolph, J.D. Fowlkes, L.F. Allard, H.M. Meyer, M.L. Simpson, P.D. Rack, Single-crystal nanowires grown via electron-beam-induced deposition, Nanotechnology 19 (2008) 345705.

[28] A. Botman, J.J.L. Mulders, C.W. Hagen, Creating pure nanostructures from electron-beam-induced deposition using purification techniques: a technology perspective, Nanotechnology 20 (2009) 372001.

[29] J. Pablo-Navarro, C. Magén, J.M. De Teresa, Purified and crystalline threedimensional electron-beam-induced deposits: the successful case of cobalt for high-performance magnetic nanowires, ACS Appl. Nano Mater. 1 (2018) 38-42.

[30] M.V. Puydinger dos Santos, A. Szkudlarek, A. Rydosz, C. Guerra-Nuñez, F. Béron, K.R. Pirota, S. Moshkalev, J.A. Diniz, I. Utke, Comparative study of postgrowth annealing of $\mathrm{Cu}(\mathrm{hfac})_{2}, \mathrm{Co}_{2}(\mathrm{CO})_{8}$ and $\mathrm{Me}_{2} \mathrm{Au}(\mathrm{acac})$ metal precursors deposited by FEBID, Beilstein J. Nanotechnol. 9 (2018) 91-101.

[31] R.R. Kunz, T.M. Mayer, Catalytic growth rate enhancement of electron beam deposited iron films, Appl. Phys. Lett. 50 (1987) 962-964.

[32] Y.M. Lau, P.C. Chee, J.T.L. Thong, V. Ng, Properties and applications of cobaltbased material produced by electron-beam-induced deposition, J. Vac. Sci. 
Technol. A 20 (2002) 1295-1302.

[33] A. Perentes, G. Sinicco, G. Boero, B. Dwir, P. Hoffmann, Focused electron beam induced deposition of nickel, J. Vac. Sci. Technol. B 25 (2007) 2228-2232.

[34] T. Lukasczyk, M. Schirmer, H.-P. Steinrück, H. Marbach, Electron-beaminduced deposition in ultrahigh vacuum: lithographic fabrication of clean iron nanostructures, Small 4 (2008) 841-846.

[35] A. Fernández-Pacheco, J.M. De Teresa, R. Córdoba, M.R. Ibarra, Magnetotransport properties of high-quality cobalt nanowires grown by focusedelectron-beam-induced deposition, J. Phys. D. Appl. Phys. 42 (2009) 055005.

[36] L.A. Rodríguez, L. Deen, R. Córdoba, C. Magén, E. Snoeck, B. Koopmans, J.M. De Teresa, Influence of the shape and surface oxidation in the magnetization reversal of thin iron nanowires grown by focused electron beam induced deposition, Beilstein J. Nanotechnol. 6 (2015) 1319-1331.

[37] S. Sangiao, C. Magén, D. Mofakhami, G. De Loubens, J.M. De Teresa, Magnetic properties of optimized cobalt nanospheres grown by focused electron beam induced deposition (FEBID) on cantilever tips, Beilstein J. Nanotechnol. 8 (2017) $2106-2115$.

[38] J. Pablo-Navarro, C. Magén, J.M. De Teresa, Three-dimensional core-shell ferromagnetic nanowires grown by focused electron beam induced deposition, Nanotechnology 27 (2016) 285302.

[39] R. Straubinger, A. Beyer, T. Ochs, W. Stolz, K. Volz, In situ thermal annealing transmission electron microscopy (TEM) investigation of III/V semiconductor heterostructures using a setup for safe usage of toxic and pyrophoric gases, 
Microsc. Microanal. 23 (2017) 751-757.

[40] R. Córdoba, R. Fernández-Pacheco, A. Fernández-Pacheco, A. Gloter, C. Magén, O. Stéphan, M.R. Ibarra, J.M. De Teresa, Nanoscale chemical and structural study of Co-based FEBID structures by STEM-EELS and HRTEM, Nanoscale Res. Lett. 6 (2011) 592.

[41] B. Liu, T. Tahmasebi, K. Ong, H. Teo, Z. Mo, J. Lam, P.K. Tan, Y. Zhao, Z. Dong, D. Houssameddine, J. Wang, J. Xue, Z. Mai, Electron radiation-induced material diffusion and nanocrystallization in nanostructured amorphous $\mathrm{CoFeB}$ thin film” Acta Mater. 161 (2018) 221-236.

[42] S. Frabboni, G.C. Gazzadi, L. Felisari, A. Spessot, Fabrication by electron beam induced deposition and transmission electron microscopic characterization of sub-10-nm freestanding Pt nanowires, Appl. Phys. Lett. 88 (2006) 213116.

[43] M. Takeguchi, M. Shimojo, K. Furuya, Fabrication of magnetic nanostructures using electron beam induced chemical vapour deposition, Nanotechnology 16 (2005) 1321-1325.

[44] T. Ichihashi, J. Fujita, M. Ishida, Y. Ochiai, In situ observation of carbonnanopillar tubulization caused by liquidlike iron particles, Phys. Rev. Lett. 92 (2004) 215702.

[45] J. Nogués, J. Sort, V. Langlais, V. Skumryev, S. Suriñach, J.S. Muñoz, M.D. Baró, Exchange bias in nanostructures, Phys. Rep. 422 (2005) 65-117.

[46] M.J. Martinez-Pérez, J. Pablo-Navarro, B. Müller, R. Kleiner, C. Magén, D. Koelle, J.M. De Teresa, J. Sesé, NanoSQUID magnetometry on individual asgrown and annealed Co nanowires at variable temperature, Nano Lett. 18 (2018) 
$7674-7682$.

[47] M. Gabureac, L. Bernau, I. Utke, G. Boero, Granular Co-C nano-Hall sensors by focused-beam-induced deposition, Nanotechnology 21 (2010) 115503.

[48] E. Berganza, M. Jaafar, M. Goiriena-Goikoetxea, J. Pablo-Navarro, A. GarcíaArribas, K. Gusliyenko, C. Magén, J.M. De Teresa, O. Chubykalo-Fesenko, A. Asenjo, Observation of hedgehog skyrmions in sub-100 nm soft magnetic nanodots, arXiv: 1803.08768.

[49] M.H. Ervin, B.M. Nichols, Electron beam induced deposition of cobalt for use as single- and multiwalled carbon nanotube growth catalyst, J. Vac. Sci. Technol. B 27 (2009) 2982-2985. 
Supplementary Material
Click here to download Supplementary Material: SI_Manuscript_LAST.pdf

Supplementary Material
Click here to download Supplementary Material: SI_Manuscript_LAST.pdf

\begin{abstract}
Click here to download Supplementary Material: SI_Manuscript_LAST.pdf
\end{abstract}

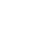

$\left(\frac{10}{2}\right.$

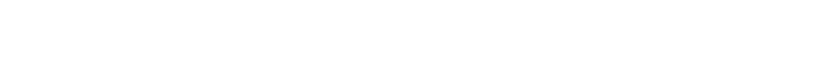

pleme
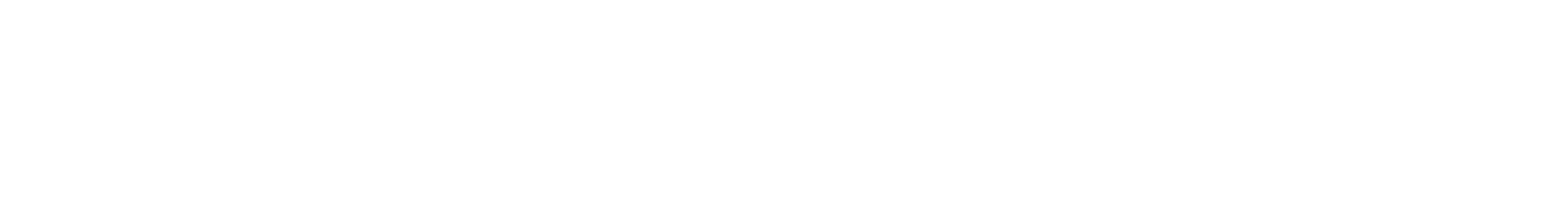

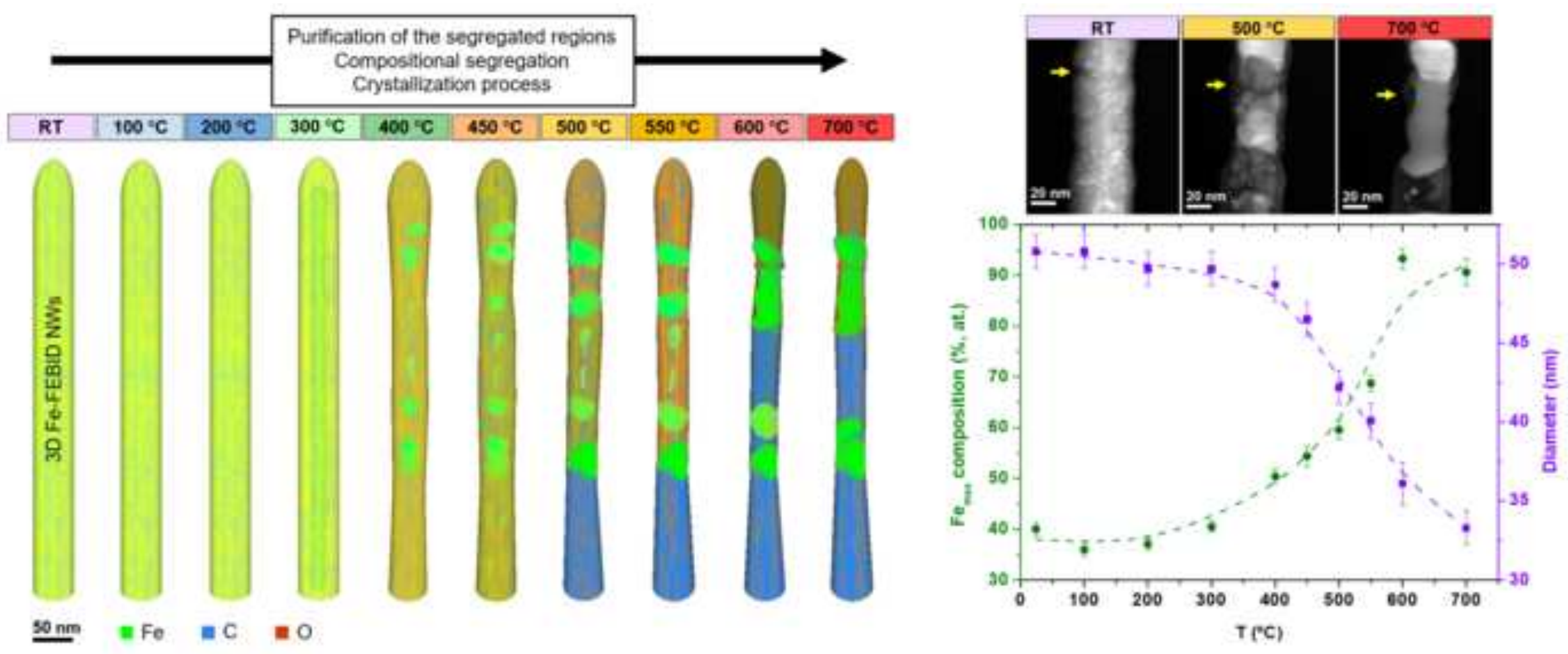
Video Still
Click here to download Video Still: VideoS1.avi Click here to download Video Stil: VideoS1.avi

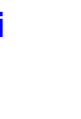

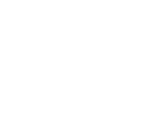

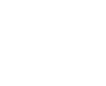
(1)

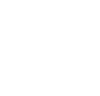

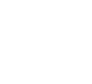
(1) (1)

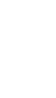
(1)

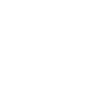

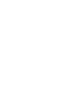

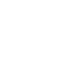

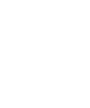
. .

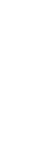
. . . .

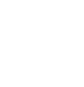

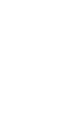

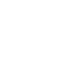

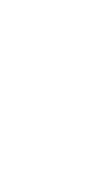

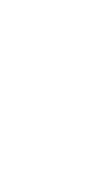

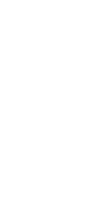
. . 列 .

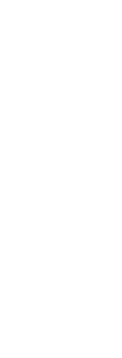

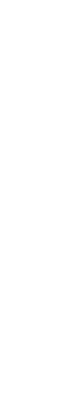

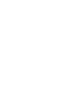

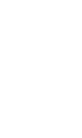

